\title{
On differentiation and homeostatic behaviours of Boolean dynamical systems ${ }^{\star}$
}

\author{
Élisabeth Remy and Paul Ruet \\ CNRS - Institut de Mathématiques de Luminy, \\ 163 avenue de Luminy, Case 907, 13288 Marseille Cedex 9 (France). \\ Email: $\{$ remy, ruet\}@iml.univ-mrs.fr
}

\begin{abstract}
We study rules proposed by the biologist $\mathrm{R}$. Thomas relating the structure of a concurrent system of interacting genes (represented by a signed directed graph called a regulatory graph) with its dynamical properties. We prove that the results in [10] are stable under projection, and this enables us to relax the assumptions under which they are valid. More precisely, we relate here the presence of a positive (resp. negative) circuit in a regulatory graph to a more general form of biological differentiation (resp. of homeostasis).
\end{abstract}

\section{Introduction}

The activity of a biological cell is to a large extent controlled by genetic regulation, which is an interacting process involving proteins and DNA (genes). We are interested here in genetic regulatory networks which abstract from the detailed genome-protein interaction by focussing on the genome and by considering interactions between genes. Such a simplification is somehow justified by the importance of DNA as a program which is present in all the cells of an organism (whereas the concentrations in proteins and in RNA transcripted from DNA vary according to the cell and the time). Genetic regulatory networks have the structure of a signed directed graph, where vertices represent genes and directed edges come equipped with a sign $(+1$ or -1$)$ and represent activatory or inhibitory effect.

This paper deals with properties relating the structure of such a concurrent system of interacting genes with its dynamics. We shall consider here discretised Boolean dynamics, ${ }^{1}$ where the activity of a gene in a specific cell is measured by the concentration of the RNA transcripted from DNA, a quantity called the expression level of the gene and assumed to be either 1 (gene expressed) or 0 (gene not expressed). Hence the state of a system of $n$ genes is modelled by an

* Corrected version of the paper published in the Transactions on Computational Systems Biology VII, Springer LNCS 4230: 153-162, 2006.

${ }^{1}$ Discrete approaches are increasingly used in biology because of the qualitative nature of most experimental data, together with a wide occurrence of non-linear regulatory relationships (e.g., combinatorial arrangements of molecular bindings, existence of cooperative or antagonist regulatory effects). 
$n$-tuple $x \in\{0,1\}^{n}$. The concurrent nature of these biological objects is clearly demonstrated for instance by a mapping to standard Petri nets $[2,9]$, of which genetic regulatory graphs can be considered as a subsystem.

The starting point of this work consists in two simple rules stated by the biologist $\mathrm{R}$. Thomas and relating the structure of regulatory graphs to their asymptotic dynamical properties [17]:

1. a necessary condition for multistability (i.e., the existence of several stable fixed points in the dynamics) is the existence of a positive circuit in the regulatory graph (the sign of a circuit being the product of the signs of its edges): this corresponds to cell differentiation processes;

2. a necessary condition for the existence of an attractive cycle in the dynamics is the existence of a negative circuit: this corresponds to homeostasis (sustained oscillatory behaviours, e.g., cell cycle or circadian rhythms).

These rules have given rise to mathematical statements and proofs mostly in a differential dynamical formalism $[8,14,4,15]$, and more recently in the discrete Boolean formalism $[1,10]$. By proving in this paper that these properties are stable under projection (in a sense that we make precise in Lemma 1), we generalise the results in [10] by showing that the existence of positive and negative circuits actually follows from weaker assumptions (Theorems 3 and 4). In the case of positive circuits for instance, the condition corresponds to a more general form of differentiation than in [10].

We do not make explicit in this introduction how regulatory graphs and dynamics are defined in terms of each other. This is done in Section 2. Let us simply observe here that instead of starting from processes which are graphs and studying their dynamics (which is typically graph rewriting, see [3] in the case of protein-protein interaction), we start here with a given dynamics and derive a regulatory graph at each point of the phase space (via a discrete form of Jacobian matrix). In particular, our approach can be used to infer circuits in regulatory networks. It is also possible to consider a fixed global "topology" of interacting genes, e.g., by taking the union of the graphs over points in the phase space, and to view our local graphs as annotations of the global one (where an interaction is "active" in a certain region of the phase space). Observe however that these more global graphs need not immediately correspond to the usual interaction graphs considered by biologists: for instance, as noticed in [16], the positive circuits occurring in $[5,7]$ are not regulatory feedback circuits, and the regulatory graphs defined in [6] are the same as ours only up to self-regulations.

We believe that the kind of properties at hand in this paper should serve as a basis to study more refined models, which could in particular take into account stochastic phenomena and metabolic pathways.

Acknowledgements. We thank Christophe Soulé and Denis Thieffry for helpful discussions. 


\section{Thomas' rules and stability under projection}

\subsection{Preliminaries}

We start by recalling here the definitions which enable to associate regulatory graphs to a dynamics. The paper is self-contained, though more details can be found in [10].

Let $n$ be a positive integer. The integers $1, \ldots, n$ denote genes. A state of the system is an $x=\left(x_{1}, \ldots, x_{n}\right) \in\{0,1\}^{n}$, where $x_{i}$ is the (discretised) expression level of gene $i: x_{i}=1$ when gene $i$ is expressed, 0 otherwise. For $\beta \in\{0,1\}$, we define $\bar{\beta}$ by $\overline{0}=1$ and $\overline{1}=0$. For $x \in\{0,1\}^{n}$ and $I \subseteq\{1, \ldots, n\}, \bar{x}^{I} \in\{0,1\}^{n}$ is defined by $\left(\bar{x}^{I}\right)_{i}=x_{i}$ for $i \notin I$ and $\left(\bar{x}^{I}\right)_{i}=\overline{x_{i}}$ for $i \in I$. When $I=\{i\}$ is a singleton, $\bar{x}^{\{i\}}$ is denoted by $\bar{x}^{i}$.

Dynamics We are interested in the dynamics of the system consisting in the $n$ interacting genes. Consider a map $f:\{0,1\}^{n} \rightarrow\{0,1\}^{n}, f(x)=\left(f_{1}(x), \ldots, f_{n}(x)\right)$. For each $x \in\{0,1\}^{n}$ and $i=1, \ldots, n, f_{i}(x)$ denotes the value to which $x_{i}$, the expression level of gene $i$, tends when the system is in state $x$. We assume that the system evolves according to the (non-deterministic) asynchronous dynamics $\left\{\left(x, \bar{x}^{i}\right)\right.$ s.t. $\left.x \in\{0,1\}^{n}, x_{i} \neq f_{i}(x)\right\}$, i.e., the expression level of only one gene is updated at each step. Other dynamics can be considered, like the (deterministic) synchronous dynamics $\left\{(x, f(x))\right.$ s.t. $\left.x \in\{0,1\}^{n}\right\}$ where all the expression levels $x_{i}$ are simultaneously updated to $f_{i}(x)$ in one step. But as argued in [10], the asynchronous one is more realistic, and Theorem 2 for instance does not hold for the synchronous one. Observe that kinetic parameters are not taken into account in the discrete approach considered in this paper; however the model could be enriched by temporal delays: this would enable to recover kinetic informations.

A cycle $($ for $f)$ is a sequence of states $\left(x^{1}, \ldots, x^{r}\right)$ such that for each $i=$ $1, \ldots, r$, the pair $\left(x^{i}, x^{i+1}\right)$ belongs to the (asynchronous) dynamics. Indices are taken here modulo $r$, i.e., $r+1=1$. A cycle $\left(x^{1}, \ldots, x^{r}\right)$ is completely described by one of its points, say $x^{1}$, and its strategy, which is the map $\varphi:\{1, \ldots, r\} \rightarrow$ $\{1, \ldots, n\}$ such that

$$
x^{i+1}={\overline{x^{i}}}^{\varphi(i)} .
$$

A cycle $\left(x^{1}, \ldots, x^{r}\right)$ with strategy $\varphi$ is said to be a trap cycle when, once in the cycle, one cannot escape any more, i.e., for all $i=1, \ldots, r$ :

$$
f\left(x^{i}\right)={\overline{x^{i}}}^{\varphi(i)} .
$$

Regulatory graphs A regulatory graph is a signed directed graph with vertex set $\{1, \ldots, n\}$, i.e., a directed graph with a sign, +1 or -1 , attached to each edge. To $f:\{0,1\}^{n} \rightarrow\{0,1\}^{n}$ and $x \in\{0,1\}^{n}$, we associate a regulatory graph $G(f)(x)$ with an edge from $j$ to $i$ when

$$
f_{i}\left(\bar{x}^{j}\right) \neq f_{i}(x),
$$


with positive sign when

$$
x_{j}=f_{i}(x),
$$

and negative sign otherwise. The intuition for the first condition is straightforward, and actually the graph underlying $G(f)(x)$ (obtained by forgetting the signs) has adjacency matrix the discrete Jacobian matrix of $f$ at $x$ defined in $[11,12]$ and recently used in [13] for proving a discrete version of Jacobian conjecture. The intuition for the second condition is that the edge is positive when the values $x_{j}$ and $f_{i}(x)$ either both increase or both decrease.

If $I \subseteq\{1, \ldots, n\}$, an $I$-circuit is a circuit $\left(n_{1}, \ldots, n_{k}\right)$ such that $n_{1}, \ldots, n_{k} \in$ $I$. If $J \subseteq I$, a $J$-circuit is clearly an $I$-circuit. The sign of a circuit is the product of the signs of its edges.

If $G$ is a regulatory graph and $I \subseteq\{1, \ldots, n\}$, the restriction of $G$ to $I$ is the regulatory graph obtained from $G$ by removing any vertex not in $I$ and any edge whose source or target is not in $I$.

Thomas' rules The following results have been proved in [10].

Theorem 1. Let $f:\{0,1\}^{n} \rightarrow\{0,1\}^{n}$. If $f$ has at least two fixed points, then there is an $x \in\{0,1\}^{n}$ such that $G(f)(x)$ has a positive circuit. More precisely, if $f$ has two fixed points $a$ and $b$, and if $I$ is such that $b=\bar{a}^{I}$, then there is an $x \in\{0,1\}^{n}$ such that $G(f)(x)$ has a positive I-circuit.

Theorem 2. If $f:\{0,1\}^{n} \rightarrow\{0,1\}^{n}$ has a trap cycle $\left(x^{1}, \ldots, x^{r}\right)$ with strategy $\varphi$, then $G(f)\left(x^{1}\right) \cup \cdots \cup G(f)\left(x^{r}\right)$ has a negative $I$-circuit with $I=\{\varphi(1), \ldots, \varphi(r)\}$.

Examples of biological situations illustrating these two kinds of dynamical properties have been studied for instance in [2]: drosophila cell cycle for an example of homeostasis and negative circuit, flowering of arabidopsis for an example of differentiation and positive circuit.

\subsection{Stability under projection}

We show that the regulatory graphs defined in Section 2.1 are stable under projection in the following sense.

Given $I \subseteq\{1, \ldots, n\}$, let $m$ be the cardinality of $I, m \leqslant n$, and let $\pi_{I}$ : $\{0,1\}^{n} \rightarrow\{0,1\}^{m}$ be the projection on $\{0,1\}^{m}$. Given such a subset $I$ of genes, there are several ways to define a dynamics on $I:$ if $f:\{0,1\}^{n} \rightarrow\{0,1\}^{n}$ and $s:\{0,1\}^{m} \rightarrow\{0,1\}^{n}$ is a section of $\pi_{I}$ (i.e., $\pi_{I} \circ s$ is the identity), let

$$
f_{I, s}=\pi_{I} \circ f \circ s:\{0,1\}^{m} \rightarrow\{0,1\}^{m} .
$$

We shall be especially interested in very specific sections, those for which genes out of $I$ are given a fixed expression level: a section $s$ is said regular when $\pi_{k} \circ s:\{0,1\}^{m} \rightarrow\{0,1\}$ is constant for each $k \notin I$.

Let us say furthermore that $I$ is compatible with $f$ when for all $x, y \in\{0,1\}^{n}$, $\pi_{I}(x)=\pi_{I}(y)$ implies $\pi_{I}(f(x))=\pi_{I}(f(y))$. In that case, all the maps $f_{I, s}$, for 
$s$ a section of $\pi_{I}$, are equal, and we may let $f_{I}:\{0,1\}^{m} \rightarrow\{0,1\}^{m}$ be their common value: $f_{I}$ is then also given by

$$
f_{I}(z)=\pi_{I}(f(x))
$$

for $x \in\{0,1\}^{n}$ any point over $z$, i.e., such that $\pi_{I}(x)=z$.

Lemma 1. Let $f:\{0,1\}^{n} \rightarrow\{0,1\}^{n}, I \subseteq\{1, \ldots, n\}$ and $z \in\{0,1\}^{m}$. If $s$ is a regular section of $\pi_{I}$, then $G\left(f_{I, s}\right)(z)$ coincides with the restriction of $G(f)(s(z))$ to I. In particular, when $I$ is compatible with $f, G\left(f_{I}\right)(z)$ is the restriction of $G(f)(x)$ to $I$ for $x \in\{0,1\}^{n}$ any point over $z$.

Proof - Let $i, j \in I$. The regulatory graph $G\left(f_{I, s}\right)(z)$ contains an edge from $j$ to $i$ if, and only if,

$$
\left(f_{I, s}\right)_{i}\left(\bar{z}^{j}\right) \neq\left(f_{I, s}\right)_{i}(z) .
$$

But $\left(f_{I, s}\right)_{i}(z)=f_{i}(s(z))$ because

$$
\pi_{i} \circ \pi_{I}=\pi_{i}
$$

for $i \in I$. On the other hand, $\left(f_{I, s}\right)_{i}\left(\bar{z}^{j}\right)=f_{i}\left(\overline{s(z)}^{j}\right)$ because, for $j \in I$, we have

$$
s\left(\bar{z}^{j}\right)=\overline{s(z)}^{j}
$$

since $s$ is regular. Hence $G\left(f_{I, s}\right)(z)$ has an edge from $j$ to $i$ if, and only if, $G(f)(s(z))$ has. The edge in $G\left(f_{I, s}\right)(z)$ is positive if, and only if,

$$
z_{j}=\left(f_{I, s}\right)_{i}(z)
$$

and the edge in $G(f)(s(z))$ is positive if, and only if,

$$
s(z)_{j}=f_{i}(s(z)) .
$$

These conditions are equivalent for $i, j \in I$.

This Lemma asserts a sort of commutation property: the regulatory graph associated to the projected dynamics is the restriction of the initial regulatory graph. Observe however that the projection does not commute with the dynamics. Indeed, let us define the asynchronous dynamics of $f_{s, I}$ : a pair $\left(z, z^{\prime}\right) \in$ $\{0,1\}^{m} \times\{0,1\}^{m}$ with $z \neq z^{\prime}$ is in the dynamics when there exists $x^{\prime} \in\{0,1\}^{n}$ such that $z^{\prime}=\pi_{I}\left(x^{\prime}\right)$ and $\left(s(z), x^{\prime}\right)$ belongs to the asynchronous dynamics of $f$. The point is that a pair $\left(x, x^{\prime}\right)$ in the dynamics of $f$ may satisfy $\pi_{I}(x)=\pi_{I}\left(x^{\prime}\right)$ (when $x^{\prime}=\bar{x}^{i}$ with $i \notin I$ ) and hence not be mapped to a pair in the dynamics of $f_{s, I}$.

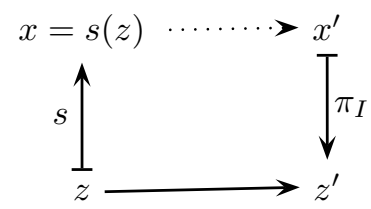


Observe that Lemma 1 does not hold when $s$ is not regular. Let indeed $f$ : $\{0,1\}^{2} \rightarrow\{0,1\}^{2}$ be given by:

$$
\begin{aligned}
& f(0,0)=(1,0) \\
& f(1,0)=(1,1) \\
& f(1,1)=(0,1) \\
& f(0,1)=(0,0),
\end{aligned}
$$

$I=\{1\}$ and $s(0)=(0,0), s(1)=(1,1)$ : then $G\left(f_{I, s}\right)(0)$ has a negative self-loop on 1 whereas $G(f)(s(0))$ consists in a positive edge from 1 to 2 and a negative edge from 2 to 1 .

When $I$ is compatible with $f$, the equivalence relation $\sim$ induced by the projection $\pi_{I}$ between states $\left(x \sim y\right.$ if, and only if, $\left.\pi_{I}(x)=\pi_{I}(y)\right)$ is a bisimulation for the asynchronous dynamics: indeed, it can be checked that if $x \sim y$ and $\left(x, x^{\prime}\right)$ is in the dynamics of $f$, then there exists $y^{\prime}$ such that $x^{\prime} \sim y^{\prime}$ and $\left(y, y^{\prime}\right)$ is in the dynamics of $f$.

Now, Lemma 1 enables us to relax the conditions of validity of Theorems 1 and 2 , as we shall see in the following sections.

\section{Disjoint stable subspaces and positive circuits}

The process of biological differentiation does not necessarily correspond to multistationarity. Consider for instance the process which controls the lysis-lysogeny decision in the bacteriophage lambda. The dynamics has a single fixed point (lysogeny) and a trap cycle (lysis): these two stable subspaces can be viewed as a differentiation phenomenon, and we would like this to imply the existence of a positive circuit (which exists indeed in the regulatory graph associated to our example, between genes $\mathrm{C} 1$ and Cro). In this Section we show that holds in general for Boolean dynamics.
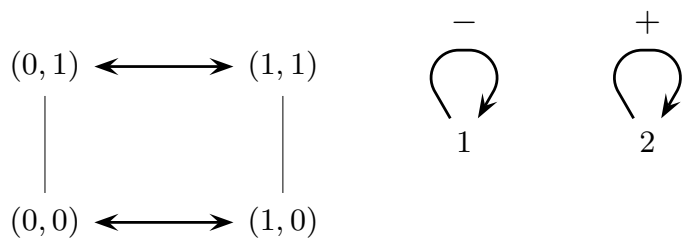

Fig. 1. On the left, a dynamics for $n=2$ with no fixed point is pictured on a framed square, and a bold arrow from state $x$ to state $\bar{x}^{i}$ means that $x_{i} \neq f_{i}(x)$. The $x$-axis carries the expression level of gene 1 and the $y$-axis the expression level of gene 2 . On the right, a positive loop on gene 2 in the (constant) regulatory graph, in accordance with Theorem 3 . 

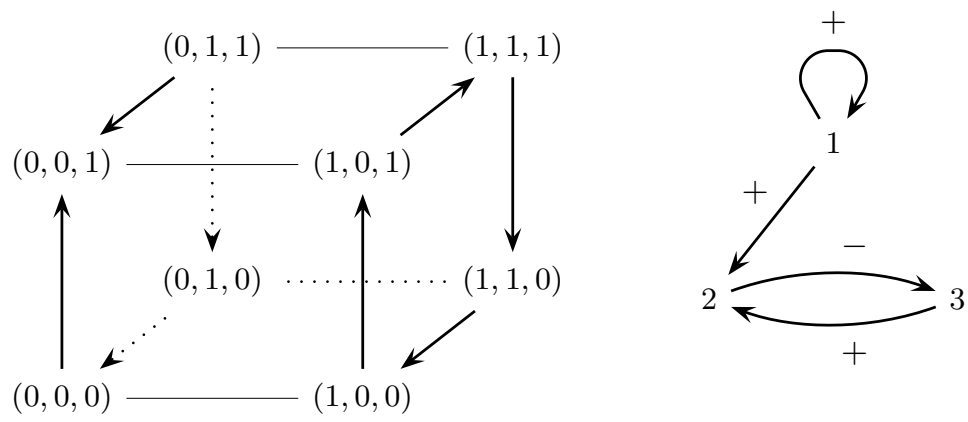

Fig. 2. On the left, a dynamics with a single fixed point $(0,0,1)$; dotted lines are only supposed to ease visualising the 3 -cube. On the right, the regulatory graph associated to the state $(1,1,1)$ has a positive loop on 1 , in accordance with Theorem 3.

Theorem 3. Let $f:\{0,1\}^{n} \rightarrow\{0,1\}^{n}, I \subseteq\{1, \ldots, n\}$ and $s$ a regular section of $\pi_{I}$. If $f_{I, s}$ has at least two fixed points, then there is an $x \in\{0,1\}^{n}$ such that $G(f)(x)$ has a positive circuit. More precisely, if $f_{I, s}$ has two fixed points a and $b$, and if $J \subseteq I$ is such that $b=\bar{a}^{J}$, then there is an $x \in\{0,1\}^{n}$ such that $G(f)(x)$ has a positive $J$-circuit.

Proof - By Theorem 1, there is a $z \in\{0,1\}^{m}$ such that $G\left(f_{I, s}\right)(z)$ has a positive $J$-circuit, and Lemma 1 suffices to conclude.

The following obvious Lemma states that multistationarity of $f_{I}$ corresponds to the existence of disjoint subspaces which are stable under $f$, clearly a more general form of biological differentiation than multistationarity.

Lemma 2. Let $f:\{0,1\}^{n} \rightarrow\{0,1\}^{n}, I \subseteq\{1, \ldots, n\}$ and $z \in\{0,1\}^{m}$. When $I$ is compatible with $f, z$ is a fixed point for $f_{I}$ if, and only if, the subspace $\pi_{I}^{-1}(z)$ is stable under $f$.

For instance, the dynamics given in Figures 1 and 2 do not have multistability, but projecting the dynamics on the $y$-coordinate $(I=\{2\} \subseteq\{1,2\}$ is compatible with $f)$ in the first case and on the $x$-coordinate $(I=\{1\} \subseteq\{1,2,3\}$ is compatible with $f$, too) in the second case, gives rise to multistability and this explains in both cases the existence of a positive circuit in the regulatory graph associated to some state.

A possible generalisation of Theorem 3 would be that positive circuits are necessary for the genuine coexistence of disjoint attractors (in our framework: disjoint sets of states which are stable under the dynamics), a conjecture which still remains to be demonstrated.

It is worth observing that this stability under projection is independent from the framework. For instance, it may be applied to the differential framework in [15]. Indeed, let $\Omega \subseteq \mathbb{R}^{n}$ be a product of open intervals in $\mathbb{R}$ and $f: \Omega \rightarrow \mathbb{R}^{n}$. 
The projection $p_{I}: \mathbb{R}^{n} \rightarrow \mathbb{R}^{m}$, where $m$ is the cardinality of $I$, is given by

$$
\left(p_{I}(x)\right)_{i}= \begin{cases}x_{i} & \text { if } i \in I, \\ 0 & \text { otherwise }\end{cases}
$$

and compatibility of $I \subseteq\{1, \ldots, n\}$ with $f$ is defined in the same way as in the Boolean case: for all $x, y \in \Omega, p_{I}(x)=p_{I}(y)$ implies $p_{I}(f(x))=p_{I}(f(y))$. In that case, we may let $f_{I}: \mathbb{R}^{m} \rightarrow \mathbb{R}^{m}$ be defined by $f_{I}(z)=p_{I}(f(x))$ for $x \in \Omega$ any point over $z$. When $f$ is continuously differentiable, C. Soulé associates to any $x \in \Omega$ a regulatory graph $G(f)(x)$ as follows: there is a positive (resp. negative) edge from $j$ to $i$ when the $(i, j)$ entry $J(f)(x)_{i, j}$ of the Jacobian matrix is positive (resp. negative).

Now, when $I$ is compatible with $f$, we have $\left(\partial\left(f_{I}\right)_{i} / \partial x_{j}\right)(z)=\left(\partial f_{i} / \partial x_{j}\right)(x)$ for $x$ any point over $z$, hence the Jacobian matrix $J\left(f_{I}\right)(z)$ is a submatrix of $J(f)(x)$ and we get the following analogous of Lemma 1: if $x \in \Omega$ is any point over $z$, then $G\left(f_{I}\right)(z)$ is the restriction of $G(f)(x)$ to $I$. This implies the following slight generalisation of Theorem 1 in [15]: if $I \subseteq\{1, \ldots, n\}$ is compatible with $f$ and $f_{I}$ has at least two nondegenerate zeros (points $a$ such that $f_{I}(a)=0$ and $\left.\operatorname{det} J\left(f_{I}\right)(a) \neq 0\right)$, then there exists $x \in \Omega$ such that $G(f)(x)$ has a positive circuit.

\section{Dynamic cycles and negative circuits}
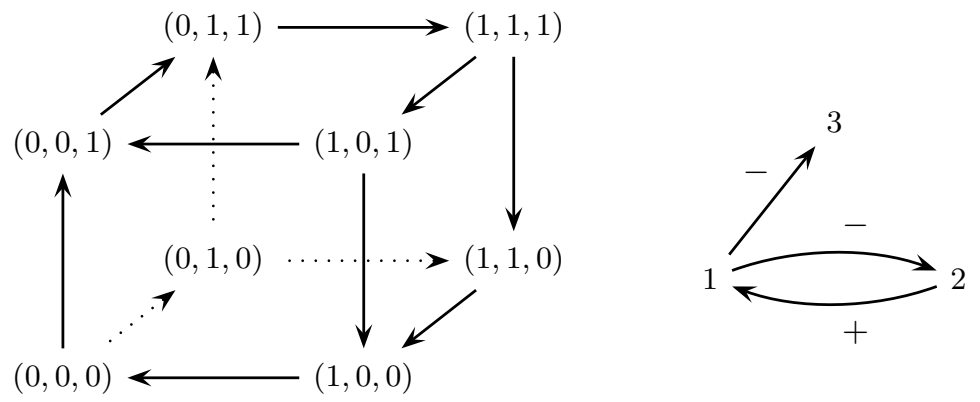

Fig. 3. On the left, a dynamics with no trap cycle. On the right, the regulatory graph associated to state $(0,0,0)$ has a negative circuit, in accordance with Theorem 4 .

Theorem 4. Let $f:\{0,1\}^{n} \rightarrow\{0,1\}^{n}, I \subseteq\{1, \ldots, n\}$ and $s$ a regular section of $\pi_{I}$. If $f_{I, s}$ has a trap cycle $\left(z^{1}, \ldots, z^{r}\right)$ with strategy $\varphi$, then

$$
G(f)\left(s\left(z^{1}\right)\right) \cup \cdots \cup G(f)\left(s\left(z^{r}\right)\right)
$$

has a negative $J$-circuit with $J=\{\varphi(1), \ldots, \varphi(r)\}$. 
Proof - By Theorem 2, $G\left(f_{I, s}\right)\left(z^{1}\right) \cup \cdots \cup G\left(f_{I, s}\right)\left(z^{r}\right)$ has a negative circuit with vertices $\varphi(1), \ldots, \varphi(r)$. Since $\varphi(1), \ldots, \varphi(r) \in I$, by Lemma 1 , this negative circuit is also in $G(f)\left(s\left(z^{1}\right)\right) \cup \cdots \cup G(f)\left(s\left(z^{r}\right)\right)$.

Figure 3 gives an example of dynamics with many dynamical cycles, none of which is a trap, hence Theorem 2 cannot be applied to infer some negative circuit. We observe that $I=\{1,2\} \subseteq\{1,2,3\}$ is compatible with $f$ : the two horizontal cycles are in parallel planes. Then by projecting on $I$, we get a trap cycle, and this explains the negative circuit involving genes 1 and 2 . In the present case, the negative circuit occurs in the regulatory graph $G(0,0,0)$ associated to a single state.
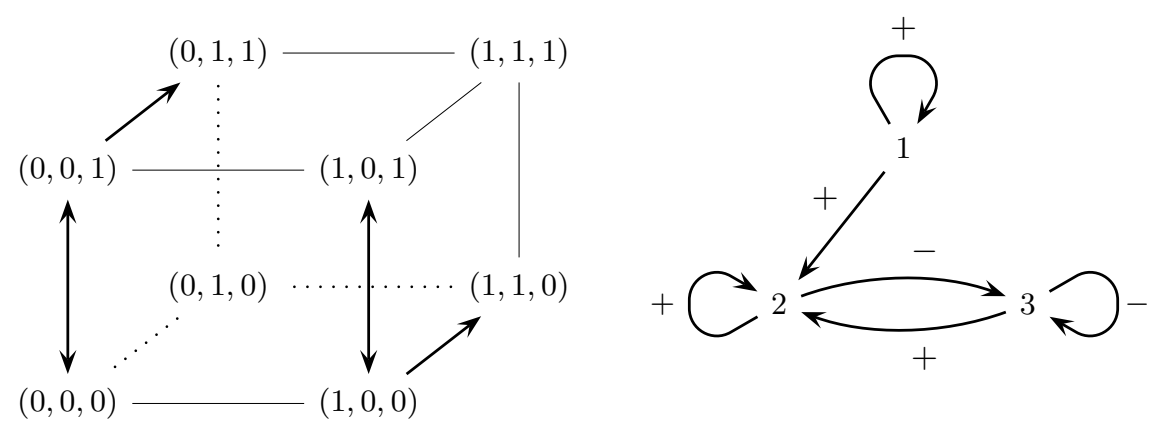

Fig. 4. On the left, a dynamics with both differentiation and homeostasis in different projections. On the right, the regulatory graph associated to state $(0,0,0)$.

A non trivial example of a dynamics with differentiation and homeostasis is given in Figure 4. On the one hand, projecting on $\{1\}$ is compatible with the dynamics and gives rise to multistationarity, whence a positive self-loop on 1 . On the other hand, projecting on $\{3\}$ and taking the following regular section:

$$
\begin{aligned}
& s(0)=(0,0,0) \\
& s(1)=(0,0,1)
\end{aligned}
$$

leads to a trap cycle between 0 and 1 , whence a negative self-loop on 3 .

\section{References}

1. J. Aracena. Modèles mathématiques discrets associés à des systèmes biologiques. Application aux réseaux de régulation génétiques. Thèse de doctorat, Université Joseph Fourier, Grenoble, 2001.

2. C. Chaouiya, E. Remy, P. Ruet, and D. Thieffry. Qualitative modelling of genetic networks: from logical regulatory graphs to standard Petri nets. In Application and Theory of Petri Nets, volume 3099 of Lecture Notes in Computer Science, pages 137-156. Springer, 2004. 
3. V. Danos and C. Laneve. Graphs for core molecular biology. In Computational Methods in Systems Biology, volume 2602 of Lecture Notes in Computer Science, pages 34-46. Springer, 2003.

4. J.-L. Gouzé. Positive and negative circuits in dynamical systems. Journal of Biological Systems, 6:11-15, 1998.

5. R. Karmakar and I. Bose. Graded and binary responses in stochastic gene expression. Technical report, arXiv:q-bio. OT/0411012, 2004.

6. F. Li, T. Long, Y. Lu, Q. Ouyang, and C. Tang. The yeast cell-cycle network is robustly designed. Proceedings of the National Academy of Sciences of the United States of America, 2004.

7. N. I. Markevich, J. B. Hoek, and B. N. Kholodenko. Signaling switches and bistability arising from multisite phosphorylation in protein kinase cascades. Journal of Cell Biology, 2004.

8. E. Plahte, T. Mestl, and S. W. Omholt. Feedback loops, stability and multistationarity in dynamical systems. Journal Biological Systems, 3:409-413, 1995.

9. É. Remy, P. Ruet, L. Mendoza, D. Thieffry, and C. Chaouiya. From logical regulatory graphs to standard Petri nets: dynamical roles and functionality of feedback circuits. In Transactions on Computational Systems Biology VII, volume 4230 of Lecture Notes in Computer Science, pages 56-72, Springer, 2006.

10. É. Remy, P. Ruet, and D. Thieffry. Graphic requirements for multistability and attractive cycles in a Boolean dynamical framework. Under review, 2005. Prépublication Institut de Mathématiques de Luminy 2005-08. Available at http://iml.univ-mrs.fr/ ruet/papiers.html.

11. F. Robert. Discrete iterations: a metric study, volume 6 of Series in Computational Mathematics. Springer, 1986.

12. F. Robert. Les systèmes dynamiques discrets, volume 19 of Mathématiques et Applications. Springer, 1995.

13. M.-H. Shih and J.-L. Dong. A combinatorial analogue of the Jacobian problem in automata networks. Advances in Applied Mathematics, 34(1):30-46, 2005.

14. E. H. Snoussi. Necessary conditions for multistationarity and stable periodicity. Journal of Biological Systems, 6:3-9, 1998.

15. C. Soulé. Graphic requirements for multistationarity. ComPlexUs, 1:123-133, 2003.

16. C. Soulé. Mathematical approaches to gene regulation and differentiation. Manuscript, 2005.

17. R. Thomas. On the relation between the logical structure of systems and their ability to generate multiple steady states and sustained oscillations. In Series in Synergetics, volume 9, pages 180-193. Springer, 1981. 